\title{
Cognitive efficiency in translation
}

Hvelplund, Kristian Tangsgaard

Published in:

Reembedding Translation Process Research

Publication date:

2016

Citation for published version (APA):

Hvelplund, K. T. (2016). Cognitive efficiency in translation. In R. Muñoz Martín (Ed.), Reembedding Translation Process Research (pp. 149-170). John Benjamins Publishing Company. Benjamins Translation Library Vol. 128 


\title{
Cognitive efficiency in translation
}

\author{
Kristian Tangsgaard Hvelplund \\ University of Copenhagen \\ bnm486@hum.ku.dk
}

\begin{abstract}
:
This article concerns the cognitive mechanisms that underlie the efficient allocation of cognitive resources during the translation process. Three indicators of efficient resource allocation are outlined and examined as correlates of translation expertise: flexibility, automaticity, and processing flow. Analyses of eye tracking and keylogging data from two groups of translatorsprofessional translators and student translators-reveal that the more experienced group performs more efficiently. Professionals exhibit greater variation in attention unit duration, indicating greater cognitive flexibility and adaptability, while analysis of pupillary data suggests a lower cognitive load on professional translators' cognitive systems. The two groups of translators exhibit different processing flow patterns. The study demonstrates that analyses of cognitive flexibility, cognitive automaticity, and processing flow are useful to determine processing efficiency in translation.
\end{abstract}

\section{Keywords:}

Cognitive translation efficiency, cognitive flexibility, cognitive automaticity, processing flow, eye tracking 


\section{Introduction}

An efficient translation process is one in which the translator exerts time and effort effectively so that cognitive resources are allocated only to those subtasks necessary to the completion of the overall translation task. This kind of processing efficiency is closely related to expertise (e.g. Ericsson and Smith 1991; Ericsson, Krampe, and Tesch-Römer 1993). The common view is that expertise and its acquisition are functions of sustained effort-i.e., deliberate practicerather than a function of talent. While talent, understood as the innate aptitude for performing well without much prior training, experience, or activity exposure, to some extent codetermines successfulness in most activities (e.g Sternberg and Ben-Zeev 2001: 302), expert performance is thought to be achievable predominantly by the continued engagement in the activity. Similarly, translators also become expert performers through deliberate practice since sustained engagement in translation activities will result in the acquisition of certain skills and strategies that are useful to the efficient execution of the translation task (e.g., Shreve 2002, 2006). The expertise paradigm has received much attention in translation studies and in translation process research, particularly in the context of specific cognitive dimensions of expertise (e.g., Jääskeläinen and Tirkkonen-Condit 1991; Shreve 2002, 2006; Englund Dimitrova 2005; Alves and Gonçalves 2007; Göpferich 2009; Muñoz Martín 2009; PACTE 2003, 2008; EhrensbergerDow and Massey 2013). The adoption of research methods from psychology and cognitive psychology, such as think-aloud protocols, cued retrospective reporting, and eye tracking, in addition to statistical methods regularly employed in psycholinguistics, have helped advance our understanding of the workings of the translator's mind. However, with respect to efficiency in the translation process, the questions of what characterises translation efficiency and if and how expertise and efficiency in translation are related have not yet been the focus of systematic investigation.

This article has two aims: the first is to outline and describe some of the cognitive mechanisms that are central to the efficient allocation of cognitive resources in translation. The outline and discussion in Section 2 focuses specifically on three interrelated but separable concepts which codetermine efficiency in translation: cognitive flexibility and cognitive automaticity, both of which originate in cognitive psychology, and processing flow, which is suggested as a conceptual and methodological tool to explore differences in translation processing. The second aim of the article is to investigate and describe actual differences between experienced and less experienced translators. Eye tracking and keylogging data are analysed to identify how experienced translators and less experienced translators differ with respect to flexibility, automaticity, and processing flow. Section 3 presents the methodological approach to this line of inquiry and Section 4 presents the findings from the three analyses, each of which considers one of the three indicators of efficiency. 


\section{Background}

Cognitive efficiency is a term used to describe broadly the mind's ability to engage effectively in activities such as planning, decision making, and problem solving with minimal cognitive effort. Therefore, efficient cognitive processing occurs when available cognitive resources are optimally invested with the aim of successful task execution. Within the field of cognitive psychology, the ability to engage effectively in planning, decision making, and problem solving involves first and foremost working memory (Baddeley and Hitch 1974; Baddeley 1986) and long-term memory (e.g., James 1890). Long-term memory is an inventory that stores the translator's knowledge, including knowledge of source language and target language (TL) vocabularies and processing strategies related to comprehension and reformulation as well as world knowledge (e.g., Gile 1995). Working memory may be seen as the executive branch of the cognitive system, which is responsible for the intentional and the unintentional selection, implementation and manipulation of knowledge from long-term memory and from the sensory organs. The translator's ability to perform efficiently is related to the cognitive system's capacity to effectively select, implement, and manipulate information during translation processing. In this article, two mechanisms are considered central to this overall translation processing efficiency: cognitive flexibility and cognitive automaticity. The flexible allocation of cognitive resources and the automation of certain subtasks during translation are assumed to codetermine overall efficiency in translation. In addition, the capacity to efficiently organise potentially attention-demanding subtasks during the translation process, as illustrated by the translator's processing flow, is thought to be related to expertise. These three aspects of cognitive efficiency in translation are explored in this section.

\subsection{Cognitive flexibility}

Generally speaking, cognitive flexibility is understood as the ability to adjust behaviour in response to changes in the surrounding environment. Related to a person's capacity to adjust the investment of mental resources in response to changing task objectives, cognitive flexibility has been defined as "the readiness with which the person's concept system changes selectively in response to appropriate environmental stimuli" (Scott 1962: 405ff). Cognitive flexibility is typically considered a component of executive functioning and it is linked to activities involving planning, problem solving, and decision making. Considered specifically within the framework of Baddeley's central executive (2007: 11ff), cognitive flexibility involves the ability: i) to focus attention on the subtasks that are most important to the execution of the overall task, ii) to switch attention between subtasks competing for cognitive resources, and iii) to divide attention more or less simultaneously between several subtasks (see also Hvelplund 2011: 45). The ability to flexibly focus, switch, and divide attention and thus allocate cognitive resources efficiently is necessary in all tasks that involve planning, problem solving, and decision making. For instance, good driving requires flexible allocation of mental resources to a constantly-changing environment where new subtasks-e.g. oncoming traffic, pedestrians crossing the street, changing traffic signals-demand the driver's attention and action. The inability to efficiently adjust resources to these new subtasks could be disastrous. 
For translation, flexible cognition is assumed to be crucial for performing efficiently, and in translation and interpreting studies the matter of flexible allocation of cognitive resources has received some attention. In his Efforts Model, Gile (1995: 186) points to a certain kind of capacity management where interpreters are "allocating and shifting processing capacity between the various Efforts." Listening and analysis, Memory, Production, and Coordination efforts compete for the interpreter's attention, and the efficiency of the interpreting process rests, in part, on the interpreter's ability to flexibly focus attention on, switch attention to, and divide attention between those efforts.

In written translation, these efforts also compete for the translator's attention, and the translator's ability to adjust allocation of resources codetermines overall processing efficiency. The ability to flexibly adjust the allocation of cognitive resources is assumed to be strongly linked to expertise since the translator through deliberate practice develops strategies that help make the most of the translator's limited pool of cognitive resources. Thus, a translator with good cognitive flexibility will focus attention for precisely as long or short a period of time as is necessary only to those subtasks which are relevant to the successful execution of the translation task. The cost of switching attention between competing subtasks will be minimal and the translator will be able to divide cognitive resources to multiple subtasks of translation simultaneously (Baddeley 2007: 11ff).

\subsection{Cognitive automaticity}

Automaticity is understood as the execution of an activity with very few cognitive resources allocated to its completion (Anderson 2000: 98). Often contrasted with automated processing, controlled processes become automated though repetition and practice, and so-called habit patterns and schemata are developed (e.g., Baddeley 2007: 120). In driving, many activities such as monitoring traffic, checking the mirrors, or maintaining the lane become automated and cognitive resources may be allocated to other tasks.

In translation research, automaticity has been discussed by some researchers, including Jääskeläinen and Tirkkonen-Condit (1991), Dragsted (2004), and Hvelplund (2011). Hvelplund (2011: 58ff) points out that, at least, two parts of the translation process can become automated: reading and typing. Through practice and repetition, the activity of typing is partly automatised (see also Spelke et al. 1976 and Salthouse 1986) and will not demand many attentional or cognitive resources. Similarly, reading is an inherently automated process. Automatic identification of meaning occurs as soon as words enter visual focus, and this process can be interrupted only when looking away from the words. So, while reading and typing rely on intentional initiation, the continuation of these activities can occur automatically. Moreover, increasing evidence suggests that possible lexical candidates in the TL are automatically identified already during source text (ST) reading (e.g., Ruiz et al. 2008; Balling et al. 2014; Schaeffer et al. 2016). In essence, target text (TT) processing is (partly) automated.

As an effect of proceduralised behaviour, the ability to automatically perform certain activities during the translation process is acquired through the prolonged and repetitive engagement in those activities. Comparing the translation processes of professional translators and student translators through think-aloud protocols, Jääskeläinen and Tirkkonen-Condit 
(1991) found that the former group spent less time on TL segments than the latter. This finding was taken as an indicator that professional translators rely more on automatic processing than student translators and it indicates that automaticity in translation is closely related to experience. The investigation reported in this article will examine automaticity in translation from a more granular cognitive perspective by investigating automaticity through pupillary data. It is hypothesised that experienced translators, to a greater extent than less experienced translators, automate parts of the translation process. Since a lighter processing load is placed on experienced translators' cognitive systems, their pupils will be overall smaller than those of less experienced translators, who have not yet developed the same habit patterns and schemata.

\subsection{Processing flow}

The third indicator of efficiency in translation is processing flow (PF). Processing flow is the sequence in which different parts of the translation are processed by the translator. The potentially attention-demanding 'macro'-elements of a translation task include, at a minimum, the ST, the TT and the keyboard, and possibly also a dictionary. Depending on the overall research goal and the nature of the translation task, the attention-demanding elements could be different and the 'macro'-elements above could be split into smaller 'micro'-elements at the segment level or even at the word level. Processing flow is expressed by the transition activity that occurs between these elements. Transition activity is measured by calculating the number of times attention shifts from one element to another. Calculation of the number and direction of transitions makes it possible to identify processing clusters, i.e., instances where there is heavy transition activity between two elements, and processing nexuses, i.e., elements which attract the majority of a translation's transition activity.

Analysis of transition activity could be used to examine a range of questions. For instance, analysis of processing flows for text genres may reveal if and how different kinds of text are processed. For specific modes of translation, processing flow analysis can be used to identify the special characteristics associated with a particular type of translating. Hvelplund (2016a) describes the processing flow in the polysemiotic activity of dubbing translation. Four macro-elements that potentially demand attention on the part of the translator include: ST, TT, Film material, and an online dictionary. TT processing was identified as a 'nexus' of transition activity as transition activity for all other elements was heaviest to and from the TT element. With respect to the question of translator expertise, comparison of processing flows of experienced translators and less experienced translators may reveal how these groups behave differently and possibly explain why professional translators often produce better translations more quickly than student translators. Intuitively, a prototypical order of sequence, or processing flow, associated with professional translator behaviour would consist first of $S T$ reading, next $T T$ typing, and finally $T T$ reading. During ST reading, meaning hypotheses are established and, possibly, aided by dictionary consultation hypotheses are confirmed. Next, during typing, the translator produces TL segments that convey the original ST meaning. The extent to which this hypothesis can be confirmed is explored in Section 4.3.

\subsection{Research questions and hypotheses}


The aim of this article is twofold. One aim is to outline and characterise some of the central cognitive features which contribute to efficient processing in translation: cognitive flexibility, cognitive automaticity, and processing flow. Based on this outline and characterisation, the second aim is to compare translation processes of experienced and less experienced translators in order to establish if and how experience correlates with efficient translation processing. The investigation relating to this second aim takes its point of departure in the general hypothesis that experienced translators expend cognitive resources more efficiently than less experienced translators. Three research questions will be examined:

- To what extent are translators able to flexibly adjust the allocation of cognitive resources in translation?

- To what extent is the translation process automated?

- What is the prototypical processing flow in translation?

Processes of experienced (professional) translators and less experienced (student) translators will be contrasted to examine specific differences in cognitive flexibility, cognitive automaticity and processing flow. Flexibility is reflected in the duration and variation in duration of individual attention units which make up the translation process (cf. Hvelplund 2011: 73). Automaticity is reflected in differences in pupil size as smaller pupils indicate relatively lighter load on the cognitive system (e.g., Hess and Polt 1964; Iqbal et al. 2005; Hvelplund 2014) and thus more automated processing. Processing flow is indicated by the number and direction of transitions for the subtasks which make up the translation process. The first two research questions will be explored also in the light of time pressure, since the study's inferential statistical analysis indicated a significant difference related to time (see Sections 4.1 and 4.2).

\section{Research design and method}

The data analysed in this article were originally collected for Hvelplund (2011). That study focused broadly on the allocation of cognitive resources in translation and explored how various factors affect resource allocation - these factors include text complexity, time pressure, and expertise. This study focusses specifically on efficiency in translation, as indicated by analyses of cognitive flexibility, automaticity and processing flow, and if and to what extent efficiency relates to translation experience.

\subsection{Participants and material}

Eye tracking and keylogging data from 24 participants were recorded. The group of less experienced translators consists of 12 participants who were students at the master's programme at the Copenhagen Business School specialising in translation between Danish and English. The participants in this group all had less than two years of translation experience. The group of experienced translators consists of 12 participants who were considered to be professional 
translators as they had more than two years of professional experience $(3-30$ years, mean $=$ 15.8 years) working as professional translators specialising in translation between Danish and English. All 24 participants have Danish as their L1 (Hvelplund 2011: 250). The size of this study's groups is considered to be sufficiently large to explore potential differences between less experienced translators and experienced translators with respect to cognitive efficiency.

The participants were instructed to translate four texts (Texts A, B, C, D) from the general news domain from (British) English into Danish. Texts A, B, and C (see the Appendix) were the texts to be analysed in the present study. They consist of 148 words, 139 words, and 132 words, respectively. Text D was presented as the first translation task to the 24 participants and served as a warm-up task intended to acclimatise them for the main task. Texts A, B, and $\mathrm{C}$ were presented to the participants in a semi-randomised order to mitigate any potential confounds related to a uniform presentation sequence (Hvelplund 2011: 84 and 86).

\subsection{Data collection and analysis}

The eye tracking and keylogging data were collected with Tobii's T60 eye tracker and its proprietary software Clearview. In addition, the keylogging software Translog (Jakobsen and Schou 1999) was used to present the ST and TT to the participants. The ST was located in the upper half of the Translog software while the TT window was located in the lower part of the programme. For the statistical analysis, $\mathrm{R}$ (version 2.11.1) was used to analyse the eye tracking and keylogging data.

Three indicators of efficiency are examined in the article's analyses of cognitive efficiency and potential processing differences between less experienced and experienced translators: 1) attention unit (AU) duration, as an indicator of cognitive flexibility; 2) pupil size, as an indicator of cognitive automaticity; and 3) transition count, as an indicator of processing flow. Following Hvelplund (2011: 73), an AU is defined as uninterrupted processing activity allocated either to the $S T$ (ST gaze activity), the $T T$ (TT gaze activity and/or typing activity) or to the ST while typing (ST gaze activity and concurrent typing). Transitions to and from an AU indicate shifts in processing activity, and the point in time at which the transition occurs is used to identify the end of one AU and the beginning of the next AU. Based on these time stamps, durations are calculated. For the pupil size measure, a pupil size mean was calculated for each AU based on the gaze samples which made up that unit. A latency effect of $120 \mathrm{~ms}$ was factored into the calculation. (See Hvelplund (ibid.) for a detailed description of the calculation of attention unit duration and pupil size.) Finally, for the processing flow analysis, the number of transitions between elements was calculated. Five attention-demanding macro-elements are defined, inspired by Hvelplund's (2016b) four types of reading in translation: ST reading, TT reading, ST reading while typing and TT reading while typing. In addition, there is the element of typing without concurrent reading, i.e., when the translator types without looking at the monitor.

\subsection{Statistical analysis}


The study's data are analysed using two types of statistical methods. Descriptive statistics are used to provide overviews of the findings with respect to attention unit duration, pupil size, and transition count. Inferential statistics are used to analyse the extent to which significant differences can be detected between less experienced and experienced translators for attention unit duration and pupil size. No inferential analysis is carried out for transition activity since this analysis consists of few $(1,440)$ items. (See also Balling and Hvelplund 2015 for a discussion of statistical methods in translation research.)

For the inferential analysis, two linear mixed-effects regression (LMER) models were fitted within the statistical environment R: one for the analysis related to attention unit duration and one for pupil size analysis. The two analyses are based on 22,947 items and 17,937 items, respectively. Both the attention unit and pupil size variables were log transformed to mitigate a positive skew in the data. The two models relate either the dependent variable $A U$ duration or the dependent variable Pupil size, respectively, with the explanatory variables Group and TimeConstraint. The former explanatory variable consists of two levels: less experienced (student) translators and experienced (professional) translators. The TimeConstraint variable, which consists of the levels TimeConstraint and NoTimeConstraint, is examined since TimeConstraint main and interaction effects in the LMER analyses are significant.

Pairwise comparisons of differences in AU duration and pupil size were carried out for different reading types. Having estimated the model, R returns $p$-values and $t$-statistics for the difference between the reference level (e.g., ST) and the other levels (i.e., ST+typing, TT, and TT+typing). Since the model returns values only for the automatically-specified reference level, the reference level had to be changed for other relevant pairwise comparisons. For the analysis of AU duration, a total of 12 pairwise comparisons were carried out and for the analysis of pupil size, a total of eight pairwise comparisons were conducted. Bonferroni correction (Baayen 2009: 106) was used to counteract the issue of multiple comparisons, and the adjusted $\alpha$-level were thus $0.05 / 12=0.0042$ and $0.05 / 8=0.00625$, respectively.

\section{Results and discussion}

In order to investigate the general hypothesis that experienced translators expend cognitive resources more efficiently than less experienced translators, this section examines the extent to which professional translators and student translators exhibit differences from each other with respect to i) the duration and variation in duration of attention units (Section 4.1), ii) the size of their pupils (Section 4.2), and iii) the number and direction of transitions (Section 4.3). Sections 4.1 and 4.2 are organised such that first overall descriptive characteristics of the data are presented as well as overall main and interaction effects of various factors thought to possibly affect cognitive resource allocation. Section 4.1 considers specifically within-group differences in more detail through pair-wise comparisons for relevant factors in order to characterise each group, whereas both Sections 4.1 and 4.2 consider between-group differences to examine how the two groups actually differ from each other with respect to AU duration and pupil size. Section 4.3 considers differences between the two groups of translators through analyses of transition count (see also Section 3.3). 


\subsection{Cognitive flexibility}

The analysis of AU aims at identifying to what extent translators are able to flexibly adjust the allocation of cognitive resources. Considering first the overall descriptive measures, mean AU duration is $909 \mathrm{~ms}$ for the group of experienced translators and $928 \mathrm{~ms}$ for the group of less experienced translators (median $=529 \mathrm{~ms}$ and $598 \mathrm{~ms}$, respectively). The means and medians suggest that less experienced translators' AUs are slightly longer than those of experienced translators. In other words, it there is some indication that less experienced translators spend more time in the ST region and the TT region before shifting attention to the other region. This might be an early indication that reading, and thus the cognitive activities associated with meaning comprehension, such as meaning hypothesis generation and confirmation, are generally performed more quickly for experienced translators than for less experienced translators. With respect to the sample standard deviation $(\sigma)$ for each group, there are also differences that might be related to experience and expertise: for the experienced translators, $\sigma$ $=1028 \mathrm{~ms}$ and for the less experienced translators, $\sigma=955 \mathrm{~ms}$. The difference in standard deviation indicates that $\mathrm{AU}$ durations for experienced translators are more spread out, i.e., that there is more variation in their durations. Experienced translators' AU durations are to a greater extent different from the mean of the sample than those of less experienced, and this could be another indication that this group of translators to a greater extent adjusts and adapts the investment of cognitive resources to fit the requirements of a current sub-task of translation.

In order to go beyond the already-presented descriptive figures and investigate to what extent these preliminary intuitions might hold, an LMER model was estimated. Table 1 presents the main and interaction effects of this analysis:

Table 1: Main effects and interaction effects of attention unit duration

\begin{tabular}{l|lllrrl}
\hline Factor(s) & $\boldsymbol{d}$ f & Sumsq & Meansq & F-value & p-value & Sig $^{\boldsymbol{i}}$ \\
\hline AttentionType & 2 & 2167.8 & 1083.9 & 1542.19 & $<.0001$ & $* * *$ \\
Group & 1 & 0.8 & 0.8 & 1.17 & .3 & \\
TimeConstraint & 2 & 19.6 & 9.8 & 13.98 & $<.0001$ & $* * *$ \\
\hline Group:AttentionType & 2 & 282.9 & 141.4 & 201.22 & $<.0001$ & $* * *$ \\
Group:TimeConstraint & 2 & 4.5 & 2.2 & 3.19 & .0413 & $*$ \\
\hline Group:AttentionType:TimeConstraint & 4 & 6.7 & 1.7 & 2.38 & .0495 & $*$ \\
\hline
\end{tabular}

Denominator degrees of freedom are 22,964 in all cases.

The main effect of the group-wise comparison indicates that there is no difference in AU duration as a function of experience $(p=.3)$; however, there are considerable differences between the two groups in all three two-way and three-way interactions.

The findings from the inferential analysis demonstrate that experienced and less experienced translators behave quite differently when processing ST, TT, and ST/TT units and when working under time pressures. These findings offer support to the intuition that experience is a factor that influences cognitive flexibility in translation. However, the present main and interaction effects analysis only demonstrates that there might be differences between or within 
the two groups of translators; it does not indicate which specific contrast(s) may drive the overall significant effects. To find out if the effects are driven by within-group differences, between-group differences or both, pairwise comparisons are conducted.

\subsubsection{Cognitive flexibility: within-group differences}

The pairwise comparisons of within-group differences are intended to reveal if and how AU duration varies within each group. The results will be helpful in determining which of the two groups exhibit greater differences with respect to AU duration and thus greater cognitive flexibility. Table 2 presents the specific contrast under investigation (Processing type / condition and $P / S$ (Professionals / Students)), descriptive mean duration in milliseconds, $p$ value and $t$-statistic:

Table 2: Within-group differences - AU duration ${ }^{\text {ii }}$

\begin{tabular}{l|llcr}
\hline Processing type / condition & P/S & Mean duration $\mathbf{m s}$ & $\boldsymbol{p}_{\text {-value }}$ ii & t-statistic $^{*}$ \\
\hline Source text AU $\leftrightarrow$ Target text AU & Prof. & $653 \mathrm{~ms} \leftrightarrow 1310 \mathrm{~ms}$ & $<.0001^{*}$ & 34.57 \\
& Stud. & $1038 \mathrm{~ms} \leftrightarrow 1019 \mathrm{~ms}$ & $<.0001^{*}$ & $7.36^{\text {iv }}$ \\
\hline No time pressure $\leftrightarrow$ Time pressure & Prof. & $925 \mathrm{~ms} \leftrightarrow 872 \mathrm{~ms}$ & .0338 & -2.12 \\
& Stud. & $980 \mathrm{~ms} \leftrightarrow 868 \mathrm{~ms}$ & $<.0001^{*}$ & -4.88 \\
\hline No time pressure & Prof. & $750 \mathrm{~ms} \leftrightarrow 1255 \mathrm{~ms}$ & $<.0001^{*}$ & 16.86 \\
Source text AU $\leftrightarrow$ Target text AU & Stud. & $1095 \mathrm{~ms} \leftrightarrow 1067 \mathrm{~ms}$ & .0017 & 3.15 \\
\hline Time pressure & Prof. & $555 \mathrm{~ms} \leftrightarrow 1319 \mathrm{~ms}$ & $<.0001^{*}$ & 22.69 \\
Source text AU $\leftrightarrow$ Target text AU & Stud. & $937 \mathrm{~ms} \leftrightarrow 975 \mathrm{~ms}$ & $<.0001^{*}$ & 5.30 \\
\hline Source text AU & Prof. & $750 \mathrm{~ms} \leftrightarrow 555 \mathrm{~ms}$ & $<.0001^{*}$ & -5.51 \\
No time pressure $\leftrightarrow$ Time pressure & Stud. & $1095 \mathrm{~ms} \leftrightarrow 937 \mathrm{~ms}$ & $<.0001^{*}$ & -4.00 \\
\hline Target text AU & Prof. & $1255 \mathrm{~ms} \leftrightarrow 1319 \mathrm{~ms}$ & .0171 & 2.39 \\
No time pressure $\leftrightarrow$ Time pressure & Stud. & $1067 \mathrm{~ms} \leftrightarrow 975 \mathrm{~ms}$ & .0054 & -2.78 \\
\hline
\end{tabular}

Experienced and less experienced translators differ on a couple of points: first, mean difference in AU duration between the six pairs of ST and TT units presented in Table 2 is considerably larger for experienced translators $(373 \mathrm{~ms})$ than for less experienced translators $(74.5 \mathrm{~ms})$. Second, the $t$-statistic means are 14.02 for the experienced translators and 4.58 for the less experienced translators, and, third, the $t$-statistic medians also suggest differences between the groups: 11.19 for the experienced translators and 4.44 for the less experienced translators. Finally, standard deviation for the $t$-statistic is considerably higher for the professionals (13.08) than for the students (1.67).

The greater variation in AU duration indicated by all measures in Table 2 demonstrate that experienced translators are overall able to more flexibly adjust the allocation of cognitive resources during translation than less experienced translators. AU duration of less experienced translators is more static and less flexible, regardless of the difference in the purpose and processing requirements underlying AUs. These within-group analyses offer support for the overall hypothesis that experienced translators allocate cognitive resources more efficiently than less experienced translators; however, the analyses do not indicate specific differences between experienced and less experienced translators. That is, the analyses do not show under 
which circumstances the two groups differ. In order to identify specific differences, betweengroup comparisons are made.

\subsubsection{Cognitive flexibility: between-group differences}

The analysis and discussion presented in Section 4.1.1 demonstrated that experienced translators exhibit greater flexibility in resource allocation than less experienced translators. The analysis in this Section will identify differences in AU duration specifically between the two groups and discuss those differences in relation to cognitive efficiency in translation and cognitive flexibility. Table 3 presents the comparisons under consideration (Processing type / condition, Professionals $\leftrightarrow$ Students) with information about mean duration in milliseconds, $p$ values, and $t$-statistics:

Table 3: Between-group differences - AU duration

\begin{tabular}{l|lcr}
\hline $\begin{array}{l}\text { Processing type / condition } \\
\text { Professionals } \leftrightarrow \text { Students }\end{array}$ & Mean duration (ms) & p-value & t-statistic \\
\hline Source text AU & & & \\
Target text AU & $653 \mathrm{~ms} \leftrightarrow 1038 \mathrm{~ms}$ & $<0.0001^{*}$ & 6.01 \\
\hline No time pressure & $1310 \mathrm{~ms} \leftrightarrow 1019 \mathrm{~ms}$ & $<0.0001^{*}$ & -3.33 \\
Time pressure & $925 \mathrm{~ms} \leftrightarrow 980 \mathrm{~ms}$ & 0.1 & 1.64 \\
\hline Source text AU + No time pressure & $750 \mathrm{~ms} \leftrightarrow 1095 \mathrm{~ms}$ & $<0.0001^{*}$ & 4.92 \\
\hline Source text AU + Time pressure & $555 \mathrm{~ms} \leftrightarrow 937 \mathrm{~ms}$ & $<0.0001^{*}$ & 5.57 \\
\hline Target text AU + No time pressure & $1255 \mathrm{~ms} \leftrightarrow 1067 \mathrm{~ms}$ & 0.05 & -1.92 \\
\hline Target text AU + Time pressure & $1319 \mathrm{~ms} \leftrightarrow 975 \mathrm{~ms}$ & $<0.0001^{*}$ & -4.11 \\
\hline
\end{tabular}

The analysis of differences between the groups show that they differ on two central points. Experienced translators' ST AUs are significantly shorter than those of less experienced translators, while TT AUs are significantly longer than those of less experienced translators. This difference persists when taking into account time pressure.

These differences are interesting since they demonstrate that resource allocation is closely related to experience. With respect to the difference in ST processing, experienced translators are able to establish and confirm meaning hypotheses more quickly than less experienced translators, who may not yet have developed a solid set of comprehension strategies to assist them in the process of identifying ST meaning. In addition, the experienced translator will, unlike the less experienced translator, economise on time and effort and only allocate as many resources to ST processing as is necessary in order to arrive at a reasonable understanding of the text. With respect to TT AUs, experienced translators allocate resources to these AUs for longer than less experienced translators for a number of possible reasons. First, it is possible that experienced translators work with larger units than the less experienced translators, however, the relatively short ST AUs do immediately not lend support to this explanation. Second, experienced translators may be more alert to the need to allocate sufficient resources to TT reformulation in order to arrive at a good translation. It should be noted that although the less experienced translator was in fact alert to this need and did allocate resources to TT processing for longer periods of time, the quality of the end result might not necessarily be better, since they may not yet have fully developed competences to produce translations of 
as good quality as experienced translators. As such, improving performance in translation is not only a matter of reallocating cognitive resources but indeed also a matter of developing comprehension and reformulation strategies.

\subsection{Cognitive automaticity}

The pupil size analysis examines to what extent experienced translators and less experienced translators differ with respect to the level of automated processing. With respect to the overall descriptive measures, mean pupil size is $3.37 \mathrm{~mm}$ for the group of experienced translators and $3.85 \mathrm{~mm}$ for the group of less experienced translators (median $=3.38 \mathrm{~mm}$ and $3.86 \mathrm{~mm}$, respectively). The means and medians show that experienced translators' pupils are overall smaller than those of less experienced translators. Based on these pupil size metrics, it seems that the activity of translating taxes less heavily on experienced translators than on less experienced translators. Assuming a relationship between pupil size and automaticity, experienced translators seem to rely more on automated processing than less experienced translators.

In order to investigate this preliminary intuition about automaticity in translation, a second LMER model was fitted. Table 4 presents the main and interaction effects of the LMER analysis:

Table 4: Main effects and interaction effects of pupil size

\begin{tabular}{l|lllrrr}
\hline Factor(s) & df & Sumsq & Meansq & F-value & p-value & Sig. \\
\hline AttentionType & 2 & 43.8 & 21.9 & 671.49 & $<0.0001$ & $* * *$ \\
Group & 1 & 0.5 & 0.5 & 14.51 & 0.0001 & $* * *$ \\
TimeConstraint & 2 & 21.4 & 10.7 & 328.54 & $<0.0001$ & $* * *$ \\
\hline Group:AttentionType & 2 & 2.1 & 1.0 & 31.78 & $<0.0001$ & $* * *$ \\
Group:TimeConstraint & 2 & 1.2 & 0.6 & 19.13 & $<0.0001$ & $* * *$ \\
\hline Group:AttentionType:TimeConstraint & 4 & 0.5 & 0.1 & 4.09 & 0.0026 & $* *$ \\
\hline
\end{tabular}

Denominator degrees of freedom are 17,883 for all cases.

The main effect of Group on pupil size indicates a highly significant difference $(p=.0001)$ between experienced and less experienced translators. The two-way and three-way interaction effects are also significant, indicating group-wise differences in pupil size for different kinds of reading (ST, TT, and ST/TT) as well as significant differences with and without time pressure. The inferential analysis does not reveal which group has the smallest overall pupil size during translation, and thus which group of the two relies more on automated processing. Pairwise comparisons are conducted to make this determination. Table 5 presents the comparison under consideration (Processing type / condition, Professionals $\leftrightarrow$ Students) with information about mean pupil size in millimetres, $p$-values, and $t$-statistics:

Table 5: Between-group differences - pupil size

\begin{tabular}{l|lll}
\hline Processing type / condition & Mean pupil size $(\mathrm{mm})$ & p-value & t-statistic \\
Professionals $\leftrightarrow$ Students & & & \\
\hline
\end{tabular}




\begin{tabular}{l|lll}
\hline Source text & $3.37 \mathrm{~mm} \leftrightarrow 3.75 \mathrm{~mm}$ & $0.0003^{*}$ & 3.61 \\
Target text & $3.41 \mathrm{~mm} \leftrightarrow 3.89 \mathrm{~mm}$ & $0.0001^{*}$ & 3.84 \\
\hline No time pressure & $3.33 \mathrm{~mm} \leftrightarrow 3.83 \mathrm{~mm}$ & $0.0001^{*}$ & 3.87 \\
Time pressure & $3.44 \mathrm{~mm} \leftrightarrow 3.87 \mathrm{~mm}$ & $0.0003^{*}$ & 3.63 \\
\hline Source text + No time pressure & $3.31 \mathrm{~mm} \leftrightarrow 3.74 \mathrm{~mm}$ & $0.0003^{*}$ & 3.66 \\
\hline Source text + Time pressure & $3.45 \mathrm{~mm} \leftrightarrow 3.76 \mathrm{~mm}$ & $<0.0001^{*}$ & 3.27 \\
\hline Target text + No time pressure & $3.37 \mathrm{~mm} \leftrightarrow 3.86 \mathrm{~mm}$ & $0.0002^{*}$ & 3.69 \\
\hline Target text + Time pressure & $3.48 \mathrm{~mm} \leftrightarrow 3.93 \mathrm{~mm}$ & $<0.0001^{*}$ & 4.12 \\
\hline
\end{tabular}

In all comparisons, pupils are significantly larger for less experienced translators than for experienced translators. This is strong indication that cognitive load is heavier for less experienced translators than for experienced translators and that experienced translators rely more on automated processing than less experienced translators. Thus, it seems that experienced translators are able to more effortlessly and efficiently allocate cognitive resources during translation. More specifically, as an effect of experienced translators' prolonged, deliberate engagement in translation tasks, they have developed more and more complex schemata that are activated in situations where familiar translation problems present themselves. Less experienced translators do not have the same extensive battery of automated, habitual patterns to fall back on, and they will have to allocate more cognitive resources to devise ad-hoc solutions to these unfamiliar translation problems.

\subsection{Processing flow}

This section presents the findings from the analysis of processing flow in translation. The aim of the analysis is to identify 'prototypical' features associated with the processing flow of experienced and less experienced translators. Two matrixes are presented: one for experienced translators (Table 6) and one for less experienced translators (Table 7). The matrixes present the number of transitions between five attention-demanding elements/activities: ST reading, ST reading while Typing, TT reading, TT reading while Typing, and Typing (see Hvelplund 2016a and Section 3.2).

Table 6: Transition matrix for experienced translators ${ }^{\mathrm{v}}$

\begin{tabular}{l|lllll|l}
\hline \multicolumn{1}{c|}{ To From } & ST reading & $\begin{array}{l}\text { ST reading } \\
+ \text { typing }\end{array}$ & TT reading & $\begin{array}{l}\text { TT reading } \\
+ \text { typing }\end{array}$ & Typing & Total \\
\hline ST reading & - & $\mathbf{1 1 1 3}$ & 778 & 244 & 121 & 2256 \\
\hline ST reading + typing & $\mathbf{1 4 1 1}$ & - & 237 & 854 & 282 & 2784 \\
\hline TT reading & 651 & 196 & - & $\mathbf{3 1 5 1}$ & 287 & 4285 \\
\hline TT reading + typing & 223 & 952 & $\mathbf{3 3 7 3}$ & - & 685 & 5233 \\
\hline Typing & 145 & 513 & 225 & 966 & - & 1849 \\
\hline Total & 2430 & 2774 & 4613 & 5215 & - & 16407 \\
\hline
\end{tabular}

The tentative hypothesis presented in Section 2.3 states that a prototypical translation flow involves first $S T$ reading (to identify ST meaning), then $T T$ typing (to reproduce ST meaning in the TL) and finally TT reading (to verify that ST meaning has been transferred as intended 
to the TL). Based on the figures in Table 6, this hypothesis can be partially confirmed for experienced translators, but with some modification: typing related transitions account for around two-thirds (65.5 percent) of the transitions after ST reading, but it is most often typing in combination with either ST reading or TT reading. Only in 5.4 percent of the cases will attention shift from ST reading and directly to Typing without some sort of concurrent reading. There is no processing nexus, i.e., one element which attracts the majority of the transition activity in a translation as in Hvelplund's (2016a) study on dubbing translation, but there are at least two processing clusters in this kind of 'traditional' translation (highlighted in bold): one involving $S T$ reading and $S T$ reading while typing and another involving $T T$ reading and $T T$ reading while typing. During the former processing cluster, attention oscillates between $S T$ reading and ST reading while typing in order to identify and confirm a ST meaning hypotheses while attempting to construct a possible TL rendition of the ST message, whereas in the latter processing cluster, a satisfactory ST meaning hypothesis has already been established, and attention oscillates between $T T$ reading and TT reading while typing in order to identify and finalise a satisfactory TL rendition of the ST message.

The question remains, however, if and to what extent experienced and less experienced translators behave similarly with respect to processing flow. Table 7 outlines the transition activity for less experienced translators.

Table 7: Transition matrix for less experienced translators ${ }^{\mathrm{vi}}$

\begin{tabular}{l|llllll}
\hline \multicolumn{1}{c}{ To } & ST reading & $\begin{array}{l}\text { ST reading } \\
\text { + typing }\end{array}$ & TT reading & $\begin{array}{l}\text { TT reading } \\
+ \text { typing }\end{array}$ & Typing & Typing \\
\hline ST reading & - & 784 & $\mathbf{1 0 0 4}$ & 180 & 133 & 2101 \\
\hline ST reading + typing & $\mathbf{1 2 6 2}$ & - & 159 & 482 & 95 & 1998 \\
\hline TT reading & 930 & 118 & - & $\mathbf{2 1 6 2}$ & 241 & 3451 \\
\hline TT reading + typing & 227 & 550 & $\mathbf{3 0 0 2}$ & - & 228 & 4007 \\
\hline Typing & 133 & 564 & 296 & 1278 & - & 2271 \\
\hline Total & 2552 & 2016 & 4461 & 4102 & 697 & 13828 \\
\hline
\end{tabular}

The two transition matrixes reveal that the two groups differ with respect to transition activity. Whereas experienced translators shift attention from ST reading to typing related activities in 65.5 percent of the cases, the corresponding figure for less experienced translators is only 52.2. It is also clear that processing clusters are different. While there is no processing nexus for less experienced translators, the transition count indicates that TT reading without typing most often succeeds ST reading; so rather than allocating cognitive resources directly to TT typing once a meaning hypothesis has been established and confirmed, the less experienced translator focuses instead resources to the TT in many cases, arguably, in order to support the process of meaning hypothesis confirmation. This processing flow is also present with the experienced translators, but not to the same extent; experienced translators are able to more quickly and efficiently arrive at a plausible and acceptable meaning hypothesis, and they need not incorporate TT reading as much into the processes associated with meaning hypothesis generation and confirmation. This explanation is in line with the explanation presented in Section 4.1 with respect to AU duration and cognitive flexibility. 


\section{Concluding remarks}

The aim of this article has been to explore and examine the notion of cognitive efficiency as a possible correlate of translation expertise. The specific focus of the article has been on cognitive flexibility, cognitive automaticity, and processing flow. The findings demonstrate significant differences in cognitive efficiency between experienced and less experienced translators. Experienced translators allocate cognitive resources more flexibly than less experienced translators and they engage in automated translation processing to a greater extent than those with less translation experience. The article suggested processing flow as a conceptual and methodological tool to investigate efficiency in translation processing. The article outlined some of the typical features associated with the processing flow of experienced translators and it found some distinctive differences between the processing flows of experienced and less experienced translators.

This investigation clearly illustrates that experience determines efficiency. It follows that flexibility and automaticity in translation (as well as an efficient processing flow) are acquired by the prolonged, deliberate engagement in translation. One potentially important aspect that has not received much attention in this article, nor in translation studies at large, is the matter of aptitude. While expertise is often considered to be an effect chiefly of practice, the relationship between expertise and aptitude has not been researched much. Future studies may explore systematically the extent to which some translators might have an innate talent for translating well without much training, and measures of flexibility, automaticity and processing flow will serve that research purpose well as a testing ground for further explorations into cognitive translation efficiency.

\section{References}

Alves, Fabio, and José Luiz Vila Real Gonçalves. 2007. "Modelling translator's competence: relevance and expertise under strutiny." In Triangulating translation: Perspectives in process oriented research, ed. by Fabio Alves, 3-24. Amsterdam: John Benjamins. http://dx.doi.org/10.1075/btl.45. 
Anderson, John R. 2000. Cognitive Psychology and its Implications (5th ed.). New York: Worth.

Baayen, Rolf Harald. 2009. Analyzing Linguistic Data: A Practical Introduction to Statistics $\begin{array}{lllll}\text { Using } & R & \text { (3rd } & \text { ed.). Cambridge: Cambridge University Press. }\end{array}$ http://dx.doi.org/10.1017/cbo9780511801686.

Baddeley, Alan David. 1986. Working Memory. Oxford: Clarendon Press.

Baddeley, Alan David. 2007. Working Memory, Thought, and Action. Oxford: Oxford University Press. http://dx.doi.org/10.1093/acprof:oso/9780198528012.001.0001.

Baddeley, Alan David, and Graham J. Hitch. 1974. "Working memory." In The Psychology of Learning and Motivation: Advances in Research and Theory, Volume 8, ed. by Gordon Bower, 47-89. New York: Academic Press.

Balling, Laura Winther, and Kristian Tangsgaard Hvelplund. 2015. "Design and Statistics in Quantitative Translation (Process) Research." In Translation as a cognitive activity, (Translation Spaces), ed. by Fabio Alves, Amparo Hurtado, Isabel Lacruz, and Ricardo Muñoz Martín, 170-187. Amsterdam and Philadelphia: John Benjamins. http://dx.doi.org/10.1075/ts.4.1.08bal.

Balling, Laura Winther, Kristian Tangsgaard Hvelplund, and Annette Camilla Sjørup. 2014. "Evidence of Parallel Processing during Translation." Meta 59 (2): 234-259. http://dx.doi.org/10.7202/1027474ar.

Dragsted, Barbara. 2004. Segmentation in Translation and Translation Memory Systems: An Empirical Investigation of Cognitive Segmentation and Effects of Integrating a TM-System into the Translation Process. Ph.D. thesis, Copenhagen Business School. Copenhagen: Samfundslitteratur.

Ehrensberger-Dow, Maureen, and Gary Massey. 2013. "Indicators of translation competence: Translators' self-concepts and the translation of titles." Journal of Writing Research 5: 103131. http://dx.doi.org/10.17239/jowr-2013.05.01.5.

Englund Dimitrova, Birgitta. 2005. Expertise and explicitation in the translation process. Amsterdam and Philadelphia: John Benjamins. http://dx.doi.org/10.1075/btl.64.

Ericsson, K. Anders, and Jacqui Smith. 1991. Toward a general theory of expertise. New York: Cambridge University Press. http://dx.doi.org/10.1002/acp.2350060511.

Ericsson, K. Anders, Ralf Th. Krampe, and Clemens Tesch-Romer. 1993. "The role of deliberate practice in the acquisition of expert performance." American Psychological Association 100: 363-406. http://dx.doi.org/10.1037/0033-295x.100.3.363. 
Gile, Daniel. 1995. Basic Concepts and Models for Interpreter and Translator Training. Amsterdam and Philadelphia: John Benjamins. http://dx.doi.org/10.1075/btl.8(1st).

Göpferich, Susanne. 2009. "Towards a model of translation competence and its acquisition: the longitudinal study TransComp." In Behind the Mind: Methods, Models and Results in Translation Process Research. (Copenhagen Studies in Language 36), ed. by Susanne Göpferich, Arnt Lykke Jakobsen, and Inger M. Mees, 11-38. Copenhagen: Samfundslitteratur.

Hess, Eckhard. H., and James M. Polt. 1964. "Pupil size in relation to mental activity in simple problem solving." Science 143: 1190-1192. http://dx.doi.org/10.1126/science.143.3611.1190.

Hvelplund, Kristian Tangsgaard. 2011. Allocation of Cognitive Resources in Translation: An eye-tracking and Key-logging Study. PhD thesis. Copenhagen: Copenhagen Business School.

Hvelplund, Kristian Tangsgaard. 2014. "Eye Tracking and Translation Process: Reflections on the Analysis and Interpretation of Eye Tracking Data." In MonTI Special Issue: Minding Translation. Con la traducción en mente, ed. by Ricardo Muñoz Martín, 221-224. Alicante: Publicaciones de la universided de Alicante. http://dx.doi.org/10.6035/monti.2014.ne1.6.

Hvelplund, Kristian Tangsgaard. 2016a. "Eye tracking and the process of dubbing translation."

Hvelplund, Kristian Tangsgaard. 2016b. "Four fundamental types of reading during translation."

Iqbal, Shamsi T., Piotr D. Adamczyk, Xianjun Sam Zheng, and Brian P. Bailey. 2005. "Towards an Index of Opportunity: Understanding Changes in Mental Workload during Task Execution." Proceedings of CHI 2005, 2nd-7th April 2005, Oregon USA: 311-320. http://dx.doi.org/10.1145/1054972.1055016.

Jääskeläinen, Riitta, and Sonja Tirkkonen-Condit. 1991. "Automated Processes in Professional vs. Non-professional Translation: a Think-aloud Protocol Study." In Empirical Research in Translation and Intercultural Studies, ed. by Sonja Tirkkonen-Condit, 89-109. Tübingen: Gunter Narr.

Jakobsen, Arnt Lykke, and Lasse Schou. 1999. "Translog Documentation Version 1.0." In Probing the Process of Translation: Methods and Results Appendix 1. (Copenhagen Studies in Language 24), ed. by Gyde Hansen, 1-36. Copenhagen: Samfundslitteratur.

James, William. 1890. Principles of Psychology. New York: Holt. http://dx.doi.org/10.1037/11059-000.

Muñoz Martín, Ricardo. 2009. "Expertise and environment in translation." Mutatis Mutandis: Revista Latinoamericana de Traducción 2 (1): 24-37. 
PACTE. 2003. "Building a Translation Competence Model." In Triangulating translation: Perspectives in process oriented research, ed. by Fabio Alves, 43-66. Amsterdam: John Benjamins. http://dx.doi.org/10.1075/btl.45.06pac.

PACTE. 2008. "First Results of a Translation Competence Experiment: Knowledge of Translation and Efficacy of the Translation Process." In Translator and Interpreter Training. Issues, Methods and Debates, ed. by John Kearns, 104-126. London: Continuum.

Ruiz, Carmen, Natalia Paredes, Pedro Macizo, and María Teresa Bajo. 2008. "Activation of Lexical and Syntactic Target Language Properties in Translation." Acta Psychologica 128: 490-500. http://dx.doi.org/10.1016/j.actpsy.2007.08.004.

Salthouse, Timothy A. 1986. "Perceptual, cognitive, and motoric aspects of transcription typing." Psychological Bulletin 99 (3): 303-319. http://dx.doi.org/10.1037/0033-2909.99.3.303.

Schaeffer, Moritz, Barbara Dragsted, Kristian Tangsgaard Hvelplund, Laura Winther Balling, and Michael Carl. 2016. "Word translation entropy in translation: evidence of early target language activation during reading for translation." In New Directions in Empirical Translation Process Research: Exploring the CRITT TPR-DB, ed. by Michael Carl, Srinivas Bangalore, and Moritz Schaeffer, 183-210. Cham: Springer. http://dx.doi.org/10.1007/978-3-319-20358-4_9.

Scott, William A. 1962. "Cognitive complexity and cognitive flexibility." Sociometry 35: 405414. http://dx.doi.org/10.2307/2785779.

Shreve, Gregory M. 2002. "Knowing translation: cognitive and experiential aspects of translation expertise from the perspective of expertise studies." In Translation Studies: Perspectives on an Emerging Discipline, ed. by Alessandra Riccardi, 150-171. Cambridge: Cambridge University Press.

Shreve, Gregory M. 2006. "The deliberate practice: Translation and expertise." Journal of Translation Studies 9 (1): 27-42.

Spelke, Elizabeth, William Hirst, and Ulric Neisser. 1976. "Skills of divided attention." Cognition 4: 215-230. http://dx.doi.org/10.1016/0010-0277(76)90018-4.

Sternberg, Robert J., and Talia Ben-Zeev. 2001. Complex cognition: the psychology of human thought. New York: Oxford University Press. http://dx.doi.org/10.1016/s0160-2896(03)000266.

\section{Appendix: Texts}




\section{Text A - Killer nurse receives four life sentences}

Hospital nurse Colin Norris was imprisoned for life today for the killing of four of his patients. 32 year old Norris from Glasgow killed the four women in 2002 by giving them large amounts of sleeping medicine. Yesterday, he was found guilty of four counts of murder following a long trial. He was given four life sentences, one for each of the killings. He will have to serve at least 30 years. Police officer Chris Gregg said that Norris had been acting strangely around the hospital. Only the awareness of other hospital staff put a stop to him and to the killings. The police have learned that the motive for the killings was that Norris disliked working with old people. All of his victims were old weak women with heart problems. All of them could be considered a burden to hospital staff.

\section{Text B - Families hit with increase in cost of living}

British families have to cough up an extra $£ 1,300$ a year as food and fuel prices soar at their fastest rate in 17 years. Prices in supermarkets have climbed at an alarming rate over the past year. Analysts have warned that prices will increase further still, making it hard for the Bank of England to cut interest rates as it struggles to keep inflation and the economy under control. To make matters worse, escalating prices are racing ahead of salary increases, especially those of nurses and other healthcare professionals, who have suffered from the government's insistence that those in the public sector have to receive below-inflation salary increases. In addition to fuel and food, electricity bills are also soaring. Five out of the six largest suppliers have increased their customers' bills.

\section{Text C - Spielberg shows Beijing red card over Darfur}

In a gesture sure to rattle the Chinese Government, Steven Spielberg pulled out of the Beijing Olympics to protest against China's backing for Sudan's policy in Darfur. His withdrawal comes in the wake of fighting flaring up again in Darfur and is set to embarrass China, which has sought to halt the negative fallout from having close ties to the Sudanese government. China, which has extensive investments in the Sudanese oil industry, maintains close links with the Government, which includes one minister charged with crimes against humanity by the International Criminal Court in The Hague. Although emphasizing that Khartoum bears the bulk of the responsibility for these ongoing atrocities, Spielberg maintains that the international community, and particularly China, should do more to end the suffering.

\footnotetext{
' One asterisk indicates that the difference is very highly significant; two asterisks indicate that the difference is highly significant; three asterisks indicate that the difference is significant. No asterisk indicates no significance. ii The pairwise comparison analysis of attention units focuses only on ST AU and TT AU since these two kinds of processing make up the majority of the processing task.

iii An asterisk indicates that the difference is significant at the Bonferroni-corrected level (see Section 3.3).

iv Although the descriptive means for student translators indicate that ST AUs are longer than their TT counterpart, the $t$-statistic in fact shows that it is the opposite: TT AUs are (slightly) longer than ST AUs.
} 
${ }^{v}$ Figures in bold font indicate the most popular destination (columns) for a transition from the five kinds of activities (rows).

vi See comment ${ }^{\mathrm{v}}$ above. 